\title{
Adults with Parkinson's Disease Undergoes Exergaming Training to Improve Balance: A Systematic Review
}

\author{
Yu-Tai Wu, Yu-Feng Wu, and Jian-Hong Ye
}

\begin{abstract}
Parkinson's disease (PD) is a neurodegenerative disease, which is affecting millions of people globally. One common problem in PD patients are postural instability which can lead to balance control impairment. PD patients' postural instability is currently one of the most difficult challenges the physician encounters when treating the patients with this long duration of disease. However, previous studies have shown that exergaming is a popular treatment for improving balance of PD patients. The purpose of this study was systematic review of exergaming training intervention and outcomes of balance among participants with PD. The search terms "exergaming," "exergames," "balance," "Parkinson's disease" "Virtual reality" and "Postural instability" were used in three electronic databases, Science direct, Pubmed and Scopus. eleven studies were systematically reviewed using the synthesis matrix. The results indicated that the exergaming training group of $P D$ participants ranged from 1 to 76. The training duration and frequency was between 20 minutes to 60 minutes, one to three times a week, for a period of 6 to 12 weeks. In terms of research methodology, most studies reviewed conducted randomized controlled trial, while two studies administered semi-experimental design and one study conducted single subject experimental design. The results of these studies indicated many benefits of exergaming training among PD participants, such as, balance, gait, fatigue and postural instability. In conclusion exergaming intervention is recommended as postural stability treatment for PD participants. This study may be used as a guide for future reference when designing exergaming balance training for future studies or rehabilitation application.
\end{abstract}

Index Terms-Postural stability, embodied learning theory, virtual reality, Wii, $\mathbf{X b o x}$.

\section{INTRODUCTION}

Parkinson's disease (PD) is a worldwide illness, with an occurrence rate of 4.5 to 19 people per 100000 populations per year [1] (World Health Organization, 2006). PD is a neurodegenerative disease, which is affecting seven million people globally [2] (Zafari, Amiri, \& Taherian, 2017) and known to effect individuals' postural instability [3] (Lee, Altmann, McFarland, \& Hass, 2016). The instability of the postural may be present in the early stage of the disease and become worse as the PD progresses [4] (Jankovic, 2008). It is found that the frequency of individuals with Parkinson's disease (PD) fall twice as much as neurotypical people [5]

Manuscript received September 14, 2019; revised December 15, 2019.

Yu-Tai $\mathrm{Wu}$ is with the Department of Physical Education, National Taiwan Normal University, Taiwan (e-mail: tarrywu2005@gmail.com).

$\mathrm{Yu}-\mathrm{Feng} \mathrm{Wu}$ is with the Graduate Institute of Sport, Leisure and Hospitality Management, National Taiwan Normal University, Taiwan (corresponding author; e-mail: garywu821822@gmail.com).

Jian-Hong Ye is with the Department of Industrial Education, National Taiwan Normal University, Taiwan (e-mail: kimpo30107@yahoo.com.tw).
(Allen, Schwarzel, \& Canning2013). Postural instability among individuals with PD not only lead to balance control impairment, an increase in fear of falling and losing balance confidence [6] (Adkin, Frank, \& Jog, 2003).

According to [1] World Health Organization (2006) PD patients face many difficulties, such as, freezing of gait, postural instability and falls, which are associated to patients' balance. In addition, PD patients face many neuromechanical impairments, such as anticipatory postural adjustments (APAs) and automatic postural reactions (APRs) that affects the movement coordination and postural instability [7] (Carpenter, Allum, Honegger, Adkin, \& Bloem, 2004; [8] Massion, 1998). Furthermore, the joint stiffness is also a common problem postural responses. In fact, these play out to be a great influence to postural influence even when standing. Postural instability is currently one of the most difficult challenges the physician encounters when treating the patients with this long duration of disease [1] (World Health Organization, 2006).

However, exergaming is a popular intervention, as can be seen, in recent years' technologies such as virtual reality and exergaming software, is increasing rapidly in neurological rehabilitation [9] (Mirelman, Maidan, \& Deutsch, 2013). Games that involves in exercising while playing for health benefits, which requires the movement of the body in order to continue with the game and helps increases physical activity levels is known as "Exergames". Convincingly, exergaming holds promises that it is more effective comparing to other programs that contain physical activity [10] (Gao, Chen, Pasco, \& Pope, 2015). Therefore, exergaming may be an implement for avoiding or decelerating the pace of losses in postural control and functional balance.

According to the embodied learning theory, which comprises learning activities with the requirement of body engagement and provides people with improved knowledge retention and learning performance. People's thought and reasoning have been argued that embodied cognition are deeply associated to the sensorimotor, at the same time as the interaction of physical environment is connected to the body [11] (Barsalou, 1999; [12] Glenberg, 2010. Moreover, embodied learning requires that the individuals have a meaningful connection, where learning is required to be associated with physical, embodied experience [13] (Merriam, 2008).

In the world today, technology is advancing its growth in a fast pace, which allowed the capabilities of interface within digital technology to gradually enable people to have connection with computers easier than ever [14] (Jacob et al., 2008). In recent years, the technology advancement has become easier for individuals to interact, taking mobile phones and tablet computers for example, these technologies 
have made it an easy movement with augmented camera views making the physical interface combining with virtual and physical settings, which is now known as "mixed reality" [15] (Milgram \& Kishino, 1994). Numerous studies have defined the extension of repertoires of interaction, and have indicated that understanding and learning the theory of embodied learning is important [16] (Bujak et al., 2013; [17] Chang, Lee, Wang, \& Chen, 2010; [18] Lindgren \& Johnson-Glenberg, 2013). [19] Resnick (2006) specified that "conceptual leverage can be led by digital technologies so that learners to accomplish embodied activities

Augmented viruality (AV) is known to improve people embodiments. The AV includes interfaces that requires interaction and devices that can bring elements to the real world, known as virtual reality environment, this environment is regarded as "mixed reality" [15] (Milgram \& Kishino, 1994; [20] Simsarian \& Akesson, 1997). This environment not only bring sense of presence, as well as giving individuals feedbacks and interactions [21] (Kim, Prestopnik, \& Biocca, 2014). Exergaming is an example of augmented virtuality, since it has virtual environment where motion devices are performed. For example, pressure sensors or devices that is able to track the motions of the body. These devices allow people to experience the real world, and the exergames allows it to respond to the gestures and physical activity of the user [22] (Won, Bailenson, \& Janssen, 2014).

The research designs, exergaming intervention designs and outcomes of previous studies all differ significantly. Thus the purpose of this study was systematic review of exergaming training intervention and outcomes of balance among participants with PD. In addition, this study aims to identify the benefits of exergaming training in improving balance among participants with PD. The results in this study may be used as a guide for future reference when designing exergaming balance training for future studies or rehabilitation application.

\section{Methods}

To conduct this systematic review, a synthesis matrix was used. This type of method is widely used in literature review, especially in health sciences. In this study, the matrix consists of columns and row, where the column is composed of variables and topics, and the rows composed of literature. A critical analysis method such as synthesis matrix is often used to develop future research.

In this study, the search terms were used "exergaming," "exergames," "balance," "Parkinson's disease" "Virtual reality" and "Postural instability". The search covered three electronic databases, Science direct, Pubmed and Scopus were searched, from 2013 to 2019, to identify relevant studies. In addition, this study only included studies in English and articles that were not theoretical, introductory or review articles. Participants without Parkinson disease and studies with traditional exercise for balance training were excluded from the search. There were four reviewers that conducted the systematic review. The first reviewer was a doctoral student from graduate institute of sport, leisure and hospitality management. The second reviewer was a professor from the same institute, while the third and fourth reviewer was doctoral student from department of physical education and department of industrial education. To ensure the accuracy of the selection of studies, four reviewer did a cross comparison while discussing together.

Several criteria were used in the selection of the articles. First all studies that used qualitative analysis were excluded in the study. Second exergaming therapy for balance was the intervention of all the studies. Finally, 82 articles were identified in the searching process. However, studies that included other types of balance therapy and other types of disease were excluded in the study. After the elimination of duplicates and irrelevant studies 11 studies were analyzed. The following flow chart of the searching process can be seen in the Fig. 1 below.

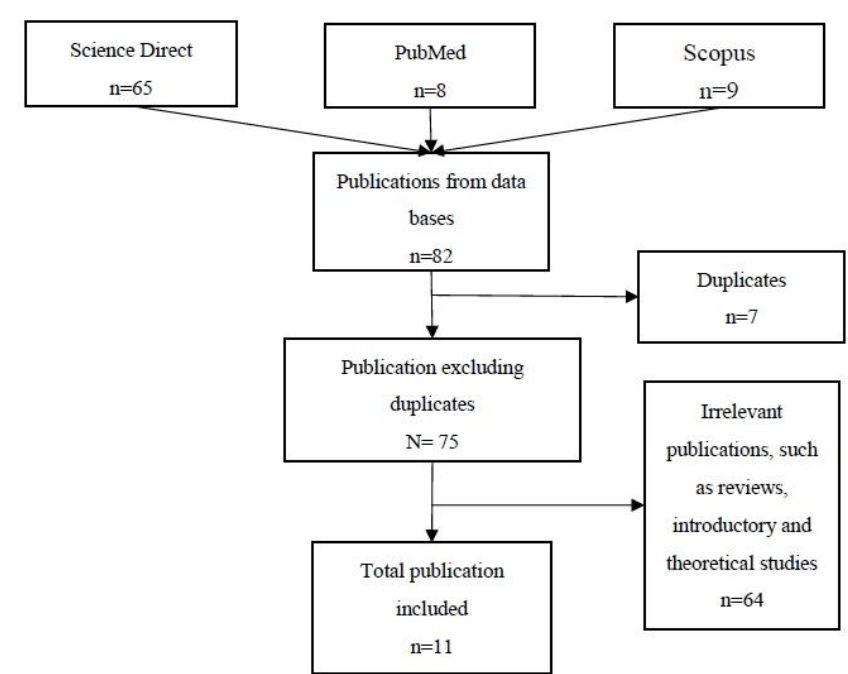

Fig. 1. Flow diagram.

\section{REsUlts}

In this study, 11 articles were used in the synthesis matrix. First, the experimental designs discussed in Table I were assessed to the type of intervention, such as participants, group types, approach and the Parkinson disease stages of the participants. Second, in Table II the results indicated the type of kits, the type of training and the duration and frequency of each experiment conducted in the 11 studies. Lastly, Table III shows outcome indicator and findings.

\section{A. Participants}

In the studies reviewed, each indicated the number of the participants that participated in their study. As can be seen, studies conducted by [23] Gandolfi et al., (2017) had the most participants accounting for 76 participants. However, there were some limitations in sample sizes in other studies [24] (Ribas et al., 2017; [25] Negrini et al., 2017; [26] Yang et al., 2016; [27] Shih et al., 2016; [28] Cracoviensia et al. 2013; [29] Mhatre, et al., 2013) all had below 30 participants except for the study conducted by [30] Henrique, Colussi, \& De Marchi, (2019) and [31] Liao et al., (2015), which had participants a little more than 30 participants. It is notable that there was one study that only had one participant [32] (Vallabhajosula, McMillion \& Freund, 2017). In addition, only 5 studies [24] (Ribas et al., 2017; [25] Negrini et al., 2017; [32] Vallabhajosula, McMillion and Freund, 2017; [28] 
Cracoviensia et al. 2013; [29] Mhatre, et al., 2013 indicated the gender of the participants, also indicated the age of the participants. However, it can be seen that more males were chosen as the participants than females for all studies. While three studies indicated the age of the participants without indicating the gender of the participants [30] (Henrique, Colussi, \& De Marchi, 2019; [33] Harris et. al., 2018; [26] Yang et al., 2016).

\section{B. Experimental Design}

8 exergaming studies have applied randomized control trial (RCT) [30] (Henrique, Colussi, \& De Marchi, 2019; [33] Harris et. al., 2018; [24] Ribas et al., 2017; [23] Gandolfi et al., 2017; [26] Yang et al., 2016; [27] Shih et al., 2016; [28] Cracoviensia et al. 2013; [29] Mhatre, et al., 2013). Randomized control trial has been indicated as strong research design because of a strong reliability [34] (Begg et al., 1996). Other two studies have applied semi-experimental design [23] (Gandolfi et al., 2017; [28] Cracoviensia et al. 2013). While one study conducted Single subject experimental design, this indicated the study only focused primarily on one participant [32] (Vallabhajosula, McMillion $\&$ Freund, 2017).

Furthermore, pre-test and post-test of exergaming training outcome were used only by two studies [27] (Shih et al., 2016; [29] Mhatre, et al., 2013) while the rest of the study compared between two groups. For example, studies from [24] Ribas et al., (2017) compared between two groups exergaming group and control group. While studies from [25] Negrini et al., 2017 compared whether 10 or 15 rehabilitation sessions of Nintando Wii Fit will have different outcome. In addition, only one study compared 3 groups virtual reality-based Wii fit exercise group, traditional exercise and control group. However, 3 studies did not have any comparisons [32] (Vallabhajosula, McMillion \& Freund, 2017; [28] Cracoviensia et al. 2013; [29] Mhatre, et al., 2013)

\section{Parkinson Disease Stage}

All studies used Hoehn \& Yahr scale to determine the stages of the Parkinson Disease among patients, except for two studies [30] (Henrique, Colussi, \& De Marchi, 2019; [25] Negrini et al., 2017). As can be seen, some studies accepted participant's with PD stage I through stage III [24] (Ribas et al., 2017; [27] Shih et al., 2016; [31] Liao et al., 2015). This suggested that the level of influence the PD patients faces will differ significantly. Thus, four studies chose participants who were more related in terms of the PD stages. Therefore, the result will be more consistent and rigorous [32] (Vallabhajosula, McMillion \& Freund, 2017; [23] Gandolfi et al., 2017; [26] Yang et al., 2016; [29] Mhatre, et al., 2013).

TABLE I: THE INTERVENTION DESCRIPTION OF THE REVIEWED STUDIES

\begin{tabular}{lllll}
\hline \multicolumn{1}{c}{ Author } & Participants & \multicolumn{1}{c}{ Group types } & \multicolumn{1}{c}{ Approach } & \multicolumn{1}{c}{ PD Stage } \\
\hline Henrique & 31 participants & experimental & Randomized & chronic \\
et al. (2019) & Age: $65-86$ & group $(\mathrm{n}=16)$ & controlled trial & ischemic stroke \\
{$[30]$} & & control group $(\mathrm{n}=$ & & $\begin{array}{l}\text { diagnosis over 6 } \\
\text { months }\end{array}$ \\
& & $15)$ & & poststroke \\
\hline Harris & & & & Hoehn and \\
et. al. (2018) & Age: 55 and & a-tDCS & controlled trial & Yahr scale \\
\hline
\end{tabular}

\begin{tabular}{|c|c|c|c|c|}
\hline Author & Participants & Group types & Approach & PD Stage \\
\hline [33] & older & $\begin{array}{l}\text { (b)exergaming + } \\
\text { sham a-tDCS } \\
\text { (c) Control group }\end{array}$ & & score 2-4 \\
\hline $\begin{array}{l}\text { Ribas } \\
\text { et al. (2017) } \\
{[24]}\end{array}$ & $\begin{array}{l}\text { Male: } 12 \\
\text { Female: } 8 \\
\text { Age: } 40-80\end{array}$ & $\begin{array}{l}\text { (a)exergaming } \\
\text { group (n=10) } \\
\text { (b) control, group } \\
(\mathrm{n}=10)\end{array}$ & $\begin{array}{l}\text { Randomized } \\
\text { controlled trial }\end{array}$ & $\begin{array}{l}\text { stage I, II or III } \\
\text { based on the } \\
\text { modified Hoehn } \\
\text { \& Yahr scale }\end{array}$ \\
\hline $\begin{array}{l}\text { Negrini } \\
\text { et al. (2017) } \\
{[25]}\end{array}$ & $\begin{array}{l}\text { Male: } 14 \\
\text { Female: } 13 \\
\text { Age: } 50-90\end{array}$ & $\begin{array}{l}\text { (a) } 10 \text { sessions } \\
\text { (b) } 15 \text { sessions }\end{array}$ & $\begin{array}{l}\text { Semi-experime } \\
\text { ntal design }\end{array}$ & $\begin{array}{l}\text { diagnosed with } \\
\mathrm{PD} \text { ( }>6 \text { months } \\
\text { from onset) }\end{array}$ \\
\hline $\begin{array}{l}\text { Vallabhajosula } \\
\text { et al. } 2017 \\
{[32]}\end{array}$ & $\begin{array}{l}\text { Male: } 1 \\
\text { Age: } 69\end{array}$ & A case study & $\begin{array}{l}\text { Single subject } \\
\text { experimental } \\
\text { design }\end{array}$ & $\begin{array}{l}\text { stage III on the } \\
\text { Modified } \\
\text { Hoehn and } \\
\text { Yahr Scale }\end{array}$ \\
\hline $\begin{array}{l}\text { Gandolfi } \\
\text { et al. (2017) } \\
{[23]}\end{array}$ & 76 participants & $\begin{array}{l}\text { (a)VR } \\
\text { telerehabilitation } \\
(n=38) \\
\text { (b)in-clinic SIBT } \\
(n=38)\end{array}$ & $\begin{array}{l}\text { Randomized } \\
\text { controlled trial }\end{array}$ & $\begin{array}{l}\text { Hoehn and } \\
\text { Yahr (H\&Y) } \\
\text { stages } 2.5 \text { to } 3\end{array}$ \\
\hline $\begin{array}{l}\text { Yang } \\
\text { et al. (2016) } \\
{[26]}\end{array}$ & $\begin{array}{l}23 \text { participants } \\
\text { Age: } 55-85\end{array}$ & $\begin{array}{l}\text { (a)home-based } \\
\text { virtual reality } \\
\text { balance training } \\
\text { (n=11) } \\
\text { (b)conventional } \\
\text { home balance } \\
\text { training (n=12) }\end{array}$ & $\begin{array}{l}\text { Randomized } \\
\text { controlled trial }\end{array}$ & $\begin{array}{l}\text { HoehneYahr } \\
\text { Stages II to III }\end{array}$ \\
\hline $\begin{array}{l}\text { Shih } \\
\text { et al. (2016) } \\
{[27]}\end{array}$ & 20 participants & $\begin{array}{l}\text { (a)Balanced based } \\
\text { exergaming (10) } \\
\text { (b)Balance } \\
\text { training (10) }\end{array}$ & $\begin{array}{l}\text { Randomized } \\
\text { controlled trial }\end{array}$ & $\begin{array}{l}\text { Hoehn and } \\
\text { Yahr } \\
\text { stages I through } \\
\text { III }\end{array}$ \\
\hline $\begin{array}{l}\text { Liao } \\
\text { et al. (2015) } \\
{[31]}\end{array}$ & 36 participants & $\begin{array}{l}\text { (a)virtual } \\
\text { reality-based Wii } \\
\text { fit exercise } \\
\text { (VRWii group) } \\
(\mathrm{n}=12) \\
\text { (b)Traditional } \\
\text { exercise }(\mathrm{n}=12) \\
(\mathrm{c}) \text { Control group } \\
(\mathrm{n}=12)\end{array}$ & $\begin{array}{l}\text { Randomized } \\
\text { controlled trial }\end{array}$ & $\begin{array}{l}\text { Hoehn and } \\
\text { Yahr stages I to } \\
\text { III }\end{array}$ \\
\hline $\begin{array}{l}\text { Cracoviensia } \\
\text { et al. (2013) } \\
{[28]}\end{array}$ & $\begin{array}{l}\text { Male: } 17 \\
\text { Female: } 7 \\
\text { Age: } 43-80\end{array}$ & N/A & $\begin{array}{l}\text { Semi-experime } \\
\text { ntal design }\end{array}$ & $\begin{array}{l}\text { Hoehn \& Yahr } \\
\text { scale }\end{array}$ \\
\hline $\begin{array}{l}\text { Mhatre } \\
\text { et al. (2013) } \\
{[29]}\end{array}$ & $\begin{array}{l}\text { Male: } 4 \\
\text { Female: } 6 \\
\text { Age: } 44-91\end{array}$ & N/A & $\begin{array}{l}\text { Randomized } \\
\text { controlled trial }\end{array}$ & $\begin{array}{l}\text { Hoehn and } \\
\text { Yahr stages } 2.5 \\
\text { or } 3\end{array}$ \\
\hline
\end{tabular}

\section{Kit and Training}

There were two studies that used different exergaming kit [30] (Henrique, Colussi, \& De Marchi, 2019; [33] Harris et al., 2018) which used Motion Rehab AVE 3D and Augmentative virtual reality software (Jintronix, Montreal, QC, Canada) with concurrent a-tDCS. The kit that were used in the two studies were not as home based kit like the other six studies, which used Wii Fit kit as the exergaming instruments [24] (Ribas et al., 2017; [25] Negrini et al., 2017 [23] Gandolfi et al., 2017; [31] Liao et al., 2015; [28] Cracoviensia et al. 2013; [29] Mhatre, et al., 2013), since Wii Fit has balance board is convenient to measure the balance of 
the participants. All training games played by the participants were all related to balance or postural stability. However, there were some limitations when using Wii Fit and Xbox Kinect kit, the sample size was not large for all the studies except for the study that used visual reality [23] (Gandolfi et $a l ., 2017)$. Thus, this can be that visual reality exergaming were able to conduct a larger sample size compared to Wii Fit and Xbox Kinect.

It is notable that the training games used were fun and challenging in these studies, which can enhance participants interest while training their balance and postural stability. Moreover, embodied learning requires that the individuals have a meaningful connection, where learning is required to be associated with physical, embodied experience [13] (Merriam, 2008). As can be seen these games have all acquired embodied experiences and that it can be a stated as "conceptual leverage" as these digital technologies can accomplish embodied activities [19] (Resnick, 2006).

\section{E. Duration and Frequency}

The duration of the exergaming training was between 20 minutes to 60 minutes while in most studies 30 minute sessions were the most common duration of the exergaming balance training for the PD participants, while 50 minute sessions were the second most among the studies. Most of the studies frequency of the exergaming training intervention were twice a week over 6 weeks and 8-week period. The longest frequency was over a 12 -week period intervention. (see Table II)

TABLE II: THE INTERVENTION DESCRIPTION OF THE REVIEWED STUDIES

\begin{tabular}{|c|c|c|c|c|}
\hline \multirow[t]{2}{*}{ Author } & \multicolumn{4}{|c|}{ Intervention } \\
\hline & Kit & Duration & Frequency & Training \\
\hline \multirow{12}{*}{$\begin{array}{l}\text { Henrique } \\
\text { et al. (2019) } \\
{[30]}\end{array}$} & Motion Rehab AVE & 30 minutes & twice a week over a & 1. flexion exercises \\
\hline & 3D & & 12 weeks period & 2. shoulder \\
\hline & & & & abduction and \\
\hline & & & & adduction \\
\hline & & & & 3. horizontal \\
\hline & & & & shoulder abduction \\
\hline & & & & and adduction \\
\hline & & & & 4. elbow extension, \\
\hline & & & & 5. wrist extension \\
\hline & & & & 6. knee flexion \\
\hline & & & & 7. hip flexion and \\
\hline & & & & abduction \\
\hline \multirow{6}{*}{$\begin{array}{l}\text { Harris } \\
\text { et al. (2018) } \\
{[33]}\end{array}$} & Augmentative & 30 minutes & twice a week over a & 1. Downhill skiing \\
\hline & virtual reality & & 12 weeks period & 2. Soccer kick \\
\hline & software (Jintronix, & & & 3. Maze \\
\hline & Montreal, QC, & & & 4. Rock climbing \\
\hline & Canada) with & & & 5. Color match \\
\hline & concurrent a-tDCS. & & & \\
\hline \multirow{7}{*}{$\begin{array}{l}\text { Ribas } \\
\text { et al. (2017) } \\
{[24]}\end{array}$} & Wii Fit & $30 \mathrm{~min}$ & Twice a week over a & 1. Basic Step \\
\hline & & sessions & 12-week period & 2. Obstacle Course \\
\hline & & & & 3. Basic Run, \\
\hline & & & & 4. Soccer Heading \\
\hline & & & & 5. Penguin Slide \\
\hline & & & & 6. Tilt City \\
\hline & & & & 7. Table Tilt. \\
\hline \multirow{3}{*}{$\begin{array}{l}\text { Negrini } \\
\text { et al. (2017) }\end{array}$} & Wii Fit & $30 \mathrm{~min}$ & Twice a week over 5 & 1. Penguin Slide \\
\hline & & & weeks (low dose) & 2. Balance Bubble \\
\hline & & & Three times a week & 3. Ski Slalom \\
\hline
\end{tabular}

\begin{tabular}{|c|c|c|c|c|}
\hline \multirow[t]{2}{*}{ Author } & \multicolumn{4}{|c|}{ Intervention } \\
\hline & Kit & Duration & Frequency & Training \\
\hline & & & over 5 weeks (high & 4. Ski Jump \\
\hline & & & dose) & 5. Table Tilt \\
\hline \multirow{5}{*}{$\begin{array}{l}\text { Vallabhajosula } \\
\text { et al. (2017) } \\
\text { [32] }\end{array}$} & treadmill walking & 30 minutes & 1 hour each week & 1.walking speed 5 to \\
\hline & and Xbox Kinect & each & 4 weeks of pre-test & $10 \%$ each week \\
\hline & exergaming & & 8 weeks of & 2.Boxing \\
\hline & & & intervention & 3.Bowling \\
\hline & & & 4 weeks of post-test & 4.Table Tennis \\
\hline \multirow{11}{*}{$\begin{array}{l}\text { Gandolfi } \\
\text { et al. (2017) }\end{array}$} & Wii Fit & 50 minutes & 3 days/week for 7 & 1. Rhythm parade \\
\hline & & & consecutive weeks & 2.Penguin Slide \\
\hline & & & & 3. Ski slalom \\
\hline & & & & 4. Snowball fight \\
\hline & & & & 5. Bird's-eye \\
\hline & & & & bulls-eye \\
\hline & & & & 6.Perfect 10 \\
\hline & & & & 7. Table tilt \\
\hline & & & & 8. Balance bubble \\
\hline & & & & 9. Tilt city \\
\hline & & & & 10. Skateboarding \\
\hline \multirow{8}{*}{$\begin{array}{l}\text { Yang } \\
\text { et al. (2016) } \\
{[26]}\end{array}$} & VR balance training & 50 minute & twice per week for 6 & 1. Star excursion \\
\hline & system & & weeks. & 2. Apple catching. \\
\hline & & & & 3. Park walking \\
\hline & & & & 4. Car racing \\
\hline & & & & 5. Cloth Washing \\
\hline & & & & 6. Cooking \\
\hline & & & & 7. Home Yoga \\
\hline & & & & 8. Table tilt \\
\hline \multirow{9}{*}{$\begin{array}{l}\text { Shih } \\
\text { et al. (2016) } \\
{[27]}\end{array}$} & Kinect sensor & 50 minute & Twice per week for & 1.Reaching task \\
\hline & & & 8-weeks & (stationary object) \\
\hline & & & & 2.Reaching task \\
\hline & & & & (moving object) \\
\hline & & & & 3.Obstacle advance \\
\hline & & & & (avoid upcoming \\
\hline & & & & object \\
\hline & & & & 4.Marching (step \\
\hline & & & & alternatively \\
\hline \multirow{10}{*}{$\begin{array}{l}\text { Liao } \\
\text { et al. (2015) } \\
{[31]}\end{array}$} & Wii Fit & 60 minute & Twice a week over 6 & 1.VRWii group Wii \\
\hline & & & weeks & fit exercised and \\
\hline & & & & treadmill training \\
\hline & & & & 2.TE group \\
\hline & & & & traditional exercise \\
\hline & & & & and treadmill \\
\hline & & & & training \\
\hline & & & & 3.Control group \\
\hline & & & & 4.Fall prevention \\
\hline & & & & education \\
\hline \multirow{3}{*}{$\begin{array}{l}\text { Cracoviensia } \\
\text { et al. } 2013 . \\
{[28]}\end{array}$} & Wii Fit & 20 minutes & Twice a day every & 1.Ski Slalom \\
\hline & & & day for 6 weeks & 2.Balance Bubble \\
\hline & & & & \\
\hline \multirow{2}{*}{$\begin{array}{l}\text { Mhatre } \\
\text { et al. (2013) }\end{array}$} & Wii Fit & 30 minutes & 3 times per week for & 1. marble tracking, \\
\hline & & & 8 weeks & 2. skiing \\
\hline [29] & & & & 3. bubble rafting \\
\hline
\end{tabular}

\section{F. Outcome Indicator}

All studies reviewed used scales and software's to measure the PD participants balance or postural stability which can be seen in Table III. The common scales used to determine balance was with Berg balance scale. Moreover, scales and measurements related to balance and functional stability 
conducted in the reviewed studies were Berg scale, Falls risk test, Stability index, Tinetti scale, Falls Efficacy Scale-International, Mini-Balance Evaluation Systems Test, Dynamic Gait Index, timed Up-and-Go test, Limits of stability (LOS), One-leg stance (OLS), Tinnet's Performance-Oriented Mobility Assessment, Activities-specifi Balance Confidence scale. Thus, there will be different findings on exergaming intervention on balance among participants with Parkinson's Disease.

\section{G. Findings}

Exergaming has showed significant balance improvement among PD participants Balance-based exergaming training gained a positive effect in the postural stability when comparing to conventional balance training [27] (Shih et al., 2016). According to [24] Ribas et al., (2017) study, it showed significant improvement in balance and fatigue after 12 weeks of exergaming in Wii Fit, while [32]Vallabhajosula, McMillion \& Freund, (2017) suggested that there was positive improvement in gait among PD participants. Moreover, it is suggested that virtual reality Wii Fit exergaming showed that exergaming improved significantly for PD participants in obstacle crossing performance and dynamic balance [31] (Liao et al., 2015). Another study mentioned that virtual reality exergaming can be an alternative to in-clinic, as it can reduce postural instability in PD patients [23] (Gandolfi et al., 2017).

Furthermore, both studies [28] Cracoviensia et al. (2013); [29] Mhatre, et al., (2013) are also an evident that exergaming intervention improves balance. exergaming holds promises that it is more effective comparing to other programs that contain physical activity [10] (Gao, Chen, Pasco, \& Pope, 2015). Thus exergaming could be a tool for preventing or slowing the pace of functional losses such as balance and postural control. The duration of sessions indicated by [25] Negrini et al., (2017) study provided evidence that 10 sessions of exercise and 15 sessions of exergaming in both groups improved the same way. Thus, this proves that the 10 sessions and 15 sessions of exergaming will have the same outcome in balance among PD participants.

TABLE III: THE INTERVENTION DESCRIPTION OF THE REVIEWED STUDIES

\begin{tabular}{lll}
\hline \multicolumn{1}{c}{ Author } & \multicolumn{1}{c}{ Outcome Indicator } & \multicolumn{1}{c}{ Findings } \\
\hline Henrique & 1. Modified Ashworth Scale & 1. both groups, patients \\
et al. (2019) & 2. Fugl-Meyer Assessment & obtained significant \\
[30] & 3. Berg Balance Scale & 2. Rehabilitation using \\
& & exergaming in post stroke \\
& & patients could be a more \\
& & efficient way and a reduction \\
& & alternative for improving \\
& & balance and upper limb motor \\
& & function. \\
& & \\
\hline Harris & 1. Limits of Stability Test & Improvements in functional, \\
et. al. (2018) & 2. static balance & neurocognitive, balance and \\
& 3. leg strength, & neurophysiological outcome \\
& 4. functional capacity, & measures will be greater and \\
& 5. cognitive task related & longer-lasting following \\
& cortical activation & concurrent exergaming and \\
\hline
\end{tabular}

\begin{tabular}{|c|c|c|}
\hline Author & Outcome Indicator & Findings \\
\hline & $\begin{array}{l}\text { 6. corticospinal excitability and inhibition } \\
\text { 7. cognitive inhibition }\end{array}$ & $\begin{array}{l}\text { a-tDCS than in those } \\
\text { receiving sham tDCS or usual } \\
\text { care. }\end{array}$ \\
\hline $\begin{array}{l}\text { Ribas } \\
\text { et al. (2017) } \\
{[24]}\end{array}$ & $\begin{array}{l}\text { 1. Berg Scale } \\
\text { 2. Fatigue Severity Scale } \\
\text { 3.(Six-Minute Walk Test) 4.(PDQ-39 5.Quality of } \\
\text { Life Questionnaire) }\end{array}$ & $\begin{array}{l}\text { 1. balance and fatigue } \\
\text { differed significantly } \\
\text { between time points. } \\
\text { 2. Two groups found no } \\
\text { differences in functional } \\
\text { exercise capacity or quality of } \\
\text { life. }\end{array}$ \\
\hline $\begin{array}{l}\text { Negrini } \\
\text { et al. (2017) } \\
{[25]}\end{array}$ & $\begin{array}{l}\text { 1. Tinetti scale } \\
\text { 2. Berg balance scale (BBS) } \\
\text { 3. Stability index (PST) } \\
\text { 4. Falls risk test (FRT) }\end{array}$ & $\begin{array}{l}\text { 1. } 10 \text { sessions significantly } \\
\text { showed improvement toward } \\
\text { balance } \\
\text { 2. No differences between } 10 \\
\text { session groups and } 15 \text { session } \\
\text { groups. }\end{array}$ \\
\hline $\begin{array}{l}\text { Vallabhajosula } \\
\text { et al. (2017) } \\
{[32]}\end{array}$ & $\begin{array}{l}\text { 1.Millisecond Software, LLC, Seattle, WA } \\
\text { 2. Mini-Balance Evaluation Systems Test } \\
\text { (Mini-BESTest) } \\
\text { 3. Falls Efficacy Scale-International (FES-I) } \\
\text { 4. 2-minute walk test }\end{array}$ & $\begin{array}{l}\text { Gait showed significant } \\
\text { improvements throughout the } \\
\text { intervention period. }\end{array}$ \\
\hline $\begin{array}{l}\text { Gandolfi } \\
\text { et al. (2017) } \\
{[23]}\end{array}$ & $\begin{array}{l}\text { 1. Dynamic Gait Index } \\
\text { 2. Berg Balance Scale }\end{array}$ & $\begin{array}{l}\text { Virtual reality can be an } \\
\text { alternative to in-clinic SIBT } \\
\text { which can reduce postural } \\
\text { instability among patients } \\
\text { with PD }\end{array}$ \\
\hline $\begin{array}{l}\text { Yang } \\
\text { et al. (2016) } \\
{[26]}\end{array}$ & $\begin{array}{l}\text { 1. Unified Parkinson's Disease Rating Scale } \\
\text { 2. Parkinson's Disease Questionnaire } \\
\text { 3.timed Up-and-Go test } \\
\text { 4. Dynamic Gait Index Parkinson's } \\
\text { Disease Questionnaire } \\
\text { 5. Berg Balance Scale. }\end{array}$ & $\begin{array}{l}\text { 1.Both groups showed } \\
\text { significant improvement in } \\
\text { timed Up-and-Go test, } \\
\text { Dynamic Gait Index and Berg } \\
\text { Balance Scale } \\
\text { 2. Two groups showed no } \\
\text { differences at posttest }\end{array}$ \\
\hline $\begin{array}{l}\text { Shih } \\
\text { et al. (2016) } \\
\text { [27] }\end{array}$ & $\begin{array}{l}\text { A. Postural stability } \\
\text { 1. One-leg stance (OLS) } \\
\text { 2. Limits of stability (LOS) } \\
\text { B. Functional balance } \\
\text { 1. timed up and go (TUG) } \\
\text { 2. Berg Balance Scale (BBS) }\end{array}$ & $\begin{array}{l}\text { Balance-based exergaming } \\
\text { training showed significant } \\
\text { improvement in postural } \\
\text { stability when comparing to } \\
\text { conventional balance training }\end{array}$ \\
\hline $\begin{array}{l}\text { Liao } \\
\text { et al. (2015) } \\
{[31]}\end{array}$ & $\begin{array}{l}\text { A. Primary outcomes } \\
\text { 1.Obstacle crossing performance } \\
\text { 2.Dynamic balance } \\
\text { B Secondary outcomes } \\
\text { 1.Sensory organization test (SOT) } \\
\text { 2.Parkinson's Disease Questionnaire (PDQ39) } \\
\text { 3.fall efficacy scale (FES-I) } \\
\text { 4.timed up and go test (TUG). }\end{array}$ & $\begin{array}{l}\text { Virtual reality with Will } \\
\text { showed significant } \\
\text { improvements in obstacle } \\
\text { crossing performance and } \\
\text { dynamic balance in PD } \\
\text { participants. }\end{array}$ \\
\hline $\begin{array}{l}\text { Cracoviensia } \\
\text { et al. 2013) } \\
{[28]}\end{array}$ & $\begin{array}{l}\text { 1. The Activities-specific Balance Confidence } \\
\text { (ABC) } \\
\text { 2. Unified Parkinson's Disease Rating } \\
\text { 3. The sit-to-stand test (STST) } \\
\text { 4. The Timed Up-and-Go (TUG) 5. 10-Meter } \\
\text { Walk Test (10MWT) } \\
\text { 6. Tinnet's Performance-Oriented Mobility }\end{array}$ & $\begin{array}{l}\text { Wii-Fit exergaming balance } \\
\text { board showed significant } \\
\text { improvement in PD patients' } \\
\text { dynamic functional balance } \\
\text { and motor disability }\end{array}$ \\
\hline
\end{tabular}




\begin{tabular}{lll}
\hline \multicolumn{1}{c}{ Author } & \multicolumn{1}{c}{ Outcome Indicator } & \multicolumn{1}{c}{ Findings } \\
\hline & Assessment (POMA) & \\
& 7. Berg-Balance Scale (BBS) & Wii Fit balance board showed \\
\hline Mhatre & 1.Berg Balance Scale & significant improvement such \\
et al. (2013) & 2.Dynamic Gait Index & as balance and gait in PD \\
{$[29]$} & 4. (Wii board) & participants. However, mood \\
& 5.Activities-specifi Balance Confidence scale & or confidence regarding \\
& 6.Geriatric Depression Scale & balance showed no \\
& & significance \\
\hline
\end{tabular}

\section{DISCUSSION}

The aim of this systematic review was to investigate exergaming training intervention and outcomes of balance among participants with PD. The systematic review identified 11 studies comparing different exergaming kits and tools and alternative intervention in PD participants [30] (Henrique, Colussi, \& De Marchi, 2019; [33] Harris et. al., 2018; [24] Ribas et al., 2017; [23] Gandolfi et al., 2017; [26] Yang et al., 2016; [27] Shih et al., 2016; [28] Cracoviensia et al. 2013; [29] Mhatre, et al., 2013; [34] Begg et al., 1996; [23] Gandolfi et al., 2017; [28] Cracoviensia et al. 2013). Embodied learning theory involves engaging the body in learning activities which can provide individuals with better learning performance and knowledge retention. Embodied cognition is deeply associated to the sensorimotor, at the same time as the interaction of physical environment is connected to the body, which is an evident that exergaming works as a rehabilitation tool for participants with PD [11] (Barsalou, 1999; [12] Glenberg, 2010.

Wii fit was found to be the more popular intervention among other studies, this could be that Wii fit provides balance board. The longest duration of training was 60 minutes [31] (Liao et al, 2015). More than half of the studies conducted a $30 \mathrm{~min}$ exergaming intervention [30] Henrique $\mathrm{et}$ al., 2019 [33] Harris et al., 2018; [24] Ribas et al., 2017; [25]Negrini et al., 2017; [32]Vallabhajosula et al., 2017; [29]Mhatre et al., 2013). However, another study results found that for as little as 20 minutes of exergaming can improve balance in PD patients [28] (Cracoviensia et al. 2013). Most of the studies frequency of the exergaming training intervention were twice a week over 6 weeks and 8 -week period. The longest frequency was over a 12 -week period intervention. [30] (Henrique, Colussi, \& De Marchi, 2019; [33] Harris et. al., 2018; [24] Ribas et al., 2017; [23] Gandolfi et al., 2017; [26] Yang et al., 2016; [27] Shih et al., 2016; [28] Cracoviensia et al. 2013; [29] Mhatre, et al., 2013; [34] Begg et al., 1996; [23] Gandolfi et al., 2017; [28] Cracoviensia et al. 2013). As can be seen these games have all acquired embodied experiences and that it can be a stated as "conceptual leverage" as these digital technologies can accomplish embodied activities [19] (Resnick, 2006).

Furthermore, the results also showed that 10 sessions and 15 session have the same effect on balance performance among PD patients [25] (Negrini et al., 2017). Exergaming training for balance positively improved the postural instability when comparing with conventional balance training [27] (Shih et al., 2016). In addition, another study found that exergaming also had positive effect improving balance and fatigue reduction in PD patients after 12 weeks of training. However, this benefit did not continue in the long-term. In conclusion, exergaming intervention is recommended as postural stability treatment for PD participants.

\section{CONCLUSION}

PD is a neurodegenerative disease, which effects millions of people globally [2] (Zafari, Amiri, \& Taherian, 2017). Which in result PD participants encounter postural instability in the early stage of the disease and become worse as the PD progresses [4] (Jankovic, 2008). As the PD progresses patients fall as much as neurotypical people [5] (Allen, Schwarzel, \& Canning2013). This becomes a problem, since postural instability are found to be a difficult challenge for physician when treating the PD patients [1] (World Health Organization, 2006). Thus exergaming can become can be an alternative to in-clinic sensory integration balance training (SIBT), as it can reduce postural instability in PD patients [23] (Gandolfi et al., 2017).

In recent years, exergaming training is a popular intervention, which is increasing rapidly in neurological rehabilitation [9] (Mirelman, Maidan, \& Deutsch, 2013). In the world today, technology is advancing its growth in a fast pace, which allowed the capabilities of interface within digital technology to gradually enable people to have connection with computers easier than ever [14] (Jacob et al., 2008). Therefore, exergaming for balance training can provide easier access for PD patients. Different kinds of technologies include interfaces that requires interaction such as Wii Fit, Xbox sensor and virtual reality that bring elements of the real world [15] (Milgram \& Kishino, 1994; [20] Simsarian \& Akesson, 1997).

\section{LiMITATIONS AND FUTURE STUdIES}

There were some limitations to this study. First, the studies identified to meet the eligible criteria was limited. Second, the review of the studies included many types of interventions, types of controls, measurement of results, and quality of methodologies. Third, considering that only published journal from three electronic databases, "Science direct", "Pubmed" and "Scopus" were used and that the search terms were only limited to "exergaming," "exergames," "balance," "Parkinson's disease" "Virtual reality" and "Postural instability". Finally, the search was limited to English publications, both publication bias and language bias can occur.

The results in this study may be used as a guide for future reference when designing exergaming balance training for future studies or rehabilitation application. As can be seen, the rehabilitation of the games and kits were games not designed specifically for adults with PD. Future studies may design games that focus on the needs of PD participants, including appropriate content, interface design and game needs. Future studies should indicate the standard measures of PD disease such as (Hoehn and Yahr ) [30] (Henrique, Colussi, \& De Marchi, 2019; [25] Negrini et al., 2017). 


\section{CONFLICT OF INTEREST}

The authors declare no conflict of interest.

\section{INTEREST AND AUTHOR CONTRIBUTION}

First author conducted the research, while second and third author analyzed the data. All of the authors wrote the paper and approved the final version of the paper.

\section{REFERENCES}

[1] World Health Organization, Neurological Disorders: Public Health Challenges, Author, CH, 2006.

[2] A. Zafari, P. Amiri, and R. Taherian, "Non-motor symptoms of Parkinson's disease and health-related quality of life: A mini-review," International Clinical Neuroscience Journal, vol. 4, no. 3, pp. 79-83, 2017.

[3] H. K. Lee, L. J. Altmann, N. McFarland, and C. J. Hass, "The relationship between balance confidence and control in individuals with Parkinson's disease," Parkinsonism \& Related Disorders, no. 26, pp. 24-28, 2016.

[4] J. Jankovic, "Parkinson's disease: Clinical features and diagnosis," Journal of Neurology, ” Neurosurgery \& Psychiatry, vol. 79, no. 4, pp. 368-376, 2008

[5] N. E. Allen, A. K. Schwarzel, and C. G. Canning, "Recurrent falls in Parkinson's disease: A systematic review," Parkinson's Disease, 2013.

[6] A. L. Adkin, J. S. Frank, and M. S. Jog, "Fear of falling and postural control in Parkinson's disease," Movement Disorders, vol. 18, no. 5, pp. 496-502, 2003

[7] M. G. Carpenter, J. H. J. Allum, F. Honegger, A. L. Adkin, and B. R. Bloem, "Postural abnormalities to multidirectional stance perturbations in Parkinson's disease," Journal of Neurology, Neurosurgery \& Psychiatry, vol. 75, no. 9, pp. 1245-1254, 2004.

[8] J. Massion, "Postural control systems in developmental perspective," Neuroscience \& Biobehavioral Reviews, vol. 22, no. 4, pp. 465-472, 1998.

[9] A. Mirelman, I. Maidan, and J. E. Deutsch, "Virtual reality and motor imagery: Promising tools for assessment and therapy in Parkinson's disease," Movement Disorders, vol. 28, no. 11, pp. 1597-1608, 2013.

[10] Z. Gao, S. Chen, D. Pasco, and Z. Pope, "A meta-analysis of active video games on health outcomes among children and adolescents," Obesity Reviews, vol. 16, no. 9, pp. 783-794, 2015.

[11] L. W. Barsalou, "Perceptions of perceptual symbols," Behavioral and Brain Sciences, vol. 22 no. 4, pp. 637-660, 1999.

[12] A. M. Glenberg, "Embodiment as a unifying perspective for psychology," Wiley Interdisciplinary Reviews: Cognitive Science, vol. 1, no. 4, pp. 586-596, 2010.

[13] S. B. Merriam, "Adult learning theory for the twenty-first century," New Directions for Adult and Continuing Education, vol. 2008, no. 119, pp. 93-98, 2008.

[14] R. J. Jacob, A. Girouard, L. M. Hirshfield, M. S. Horn, O. Shaer, E. T. Solovey, and J. Zigelbaum, "Reality-based interaction: a framework for post-WIMP interfaces," in Proc. the SIGCHI conference on Human Factors in Computing Systems, ACM, Chicago, pp. 201-210, April 2008.

[15] P. Milgram and F. Kishino, "A taxonomy of mixed reality visual displays," IEICE Transactions on Information and Systems, vol. 77, no. 12, pp. 1321-1329, 1994.

[16] K. R. Bujak, I. Radu, R. Catrambone, B. Macintyre, R. Zheng, and G. Golubski, "A psychological perspective on augmented reality in the mathematics classroom," Computers \& Education, no. 68, pp. 536-544, 2013.

[17] C. W. Chang, J. H. Lee, C. Y. Wang, and G. D. Chen, "Improving the authentic learning experience by integrating robots into the mixed-reality environment," Computers \& Education, vol. 55 no. 4, pp. 1572-1578, 2010.

[18] R. Lindgren and M. Johnson-Glenberg, "Emboldened by embodiment: Six precepts for research on embodied learning and mixed reality," Educational Researcher, vol. 42 no. 8, pp. 445-452, 2013.

[19] M. Resnick, "Computer as paint brush: Technology, play, and the creative society," Play= Learning: How Play Motivates and Enhances Children's Cognitive and Social-Emotional Growth, Oxford University Press, 2006, ch. 10, pp. 192-208.

[20] K. T. Simsarian and K. P. Akesson, "Windows on the world: An example of augmented virtuality," 1997.
[21] S. Y. S. Kim, N. Prestopnik, and F. A. Biocca, "Body in the interactive game: How interface embodiment affects physical activity and health behavior change," Computers in Human Behavior, no. 36, pp. 376-384, 2014.

[22] A. S. Won, J. N. Bailenson, and J. H. Janssen, “Automatic detection of nonverbal behavior predicts learning in dyadic interactions," IEEE Transactions on Affective Computing, vol. 5 no. 2, pp. 112-125, 2014.

[23] M. Gandolfi, C. Geroin, E. Dimitrova, P. Boldrini, A. Waldner, S. Bonadiman, and C. Bosello, "Virtual reality telerehabilitation for postural instability in Parkinson's disease: A multicenter, single-blind randomized, controlled trial," BioMed Research International, 2017.

[24] C. G. Ribas, L. A. Silva, M. R. Corrêa, H. G. Teive, and S. Valderramas, "Effectiveness of exergaming in improving functional balance, fatigue and quality of life in Parkinson's disease: A pilot randomized controlled trial," Parkinsonism \& Related Disorders, no. 38, pp. 13-18, 2017.

[25] S. Negrini, L. Bissolotti, A. Ferraris, F. Noro, M. D. Bishop, and J. H. Villafañe, "Nintendo Wii Fit for balance rehabilitation in patients with Parkinson's disease: A comparative study," Journal of Bodywork and Movement Therapies, vol. 2, no. 1, pp. 117-123, 2017.

[26] W. C. Yang, H. K. Wang, R. M. Wu, C. S. Lo, and K. H. Lin, "Home-based virtual reality balance training and conventional balance training in Parkinson's disease: a randomized controlled trial," Journal of the Formosan Medical Association, vol. 115, no. 9, pp. 734-743, 2016.

[27] M. C. Shih, R. Y. Wang, S. J. Cheng, and Y. R. Yang, "Effects of a balance-based exergaming intervention using the Kinect sensor on posture stability in individuals with Parkinson's disease: A single-blinded randomized controlled trial," Journal of Neuroengineering and Rehabilitation, vol. 13, no. 1, pp. 78, 2016.

[28] F. M. Cracoviensia, T. Zalecki, A. G. N. I. E. S. Z. K. A. Gorecka-Mazur, W. Pietraszko, A. D. Surowka, P. Novak, and A. N. N. A. Krygowska-Waja, "Visual feedback training using WII Fit improves balance in Parkinson's disease," Folia Medica Cracoviensia, vol. 53, no. 1, pp. 65-78, 2013.

[29] P. V. Mhatre, I. Vilares, S. M. Stibb, M. V. Albert, L. Pickering, C. M. Marciniak, and S. Toledo, "Wii fit balance board playing improves balance and gait in Parkinson disease," $P M \& R$, vol. 5, no. 9, pp. 769-777, 2013.

[30] P. P. Henrique, E. L. Colussi, and A. C. De Marchi, "Effects of exergame on patients' balance and upper limb motor function after stroke: A randomized controlled trial," Journal of Stroke and Cerebrovascular Diseases, 2019.

[31] Y. Y. Liao, Y. R. Yang, S. J. Cheng, Y. R. Wu, J. L. Fuh, and R. Y. Wang, "Virtual reality-based training to improve obstacle-crossing performance and dynamic balance in patients with Parkinson's disease," Neurorehabilitation and Neural Repair, vol. 29, no. 7, pp. 658-667, 2015.

[32] S. Vallabhajosula, A. K. McMillion, and J. E. Freund, "The effects of exergaming and treadmill training on gait, balance, and cognition in a person with Parkinson's disease: A case study," Physiotherapy Theory and Practice, vol. 33, no. 12, pp. 920-931, 2017.

[33] D. M. Harris, T. Rantalainen, M. Muthalib, L. Johnson, R. L. Duckham, S. T. Smith, and W. P. Teo, "Concurrent exergaming and transcranial direct current stimulation to improve balance in people with Parkinson's disease: study protocol for a randomized controlled trial," Trials, vol. 19 no. 1, p. $387,2018$.

[34] C. Begg, M. Cho, S. Eastwood, R. Horton, D. Moher, I. Olkin, and D. F. Stroup, "Improving the quality of reporting of randomized controlled trials: the CONSORT statement," Jama, vol. 276, no. 8, pp. 637-639, 1996.

Copyright (C) 2020 by the authors. This is an open access article distributed under the Creative Commons Attribution License which permits unrestricted use, distribution, and reproduction in any medium, provided the original work is properly cited (CC BY 4.0).

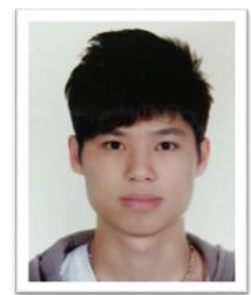

Yu-Tai Wu is a PhD student at the Department of Physical Education, National Taiwan Normal University. Mr. Wu's main research interest includes sport marketing, game-based learning, esport and sport management. 


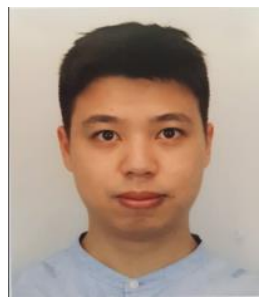

Yu-Feng $\mathbf{~ W u}$ is a $\mathrm{PhD}$ student at the Graduate Institute of Sport, Leisure and Hospitality Management, National Taiwan Normal University. Mr. Wu's main research interest includes organizational behavior, consumer behavior, sport wearables and game-based learning.

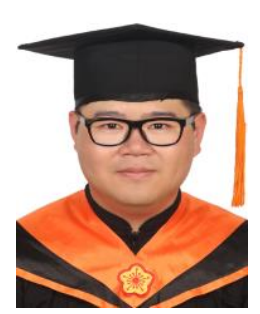

Jian-Hong Ye is a PhD student at the Department of Industrial Education, National Taiwan Normal University. He serves as research assistant at the Department of Industrial Education, National Taiwan Normal University.

Mr. Ye's main research interest includes digital learning, game-based learning, design education and vocational education. 